\title{
WI - Call for Papers Heft 1/2015
}

\section{Advanced Enterprise Modeling}

DOI 10.1007/s11576-013-0383-1

\section{Die Autoren}

Associate Prof. Jelena Zdravkovic
Associate Prof. Janis Stirna
Stockholm University
16440 Kista
Sweden
jelenaz@dsv.su.se
js@dsv.su.se

Prof. Marite Kirikova

Riga Technical University

Riga

Latvia

marite.kirikova@cs.rtu.Iv

Prof. Dimitris Karagiannis ( $\varangle$ )

Universität Wien

Wien

Österreich

dk@dke.univie.ac.at

\section{Prof. Robert Winter}

Universität St. Gallen

St. Gallen

Schweiz

robert.winter@unisg.ch

Online publiziert: 2013-08-23

This article is also available in English via http://www.springerlink.com and http://www.bise-journal.org: Zdravkovic J, Stirna J, Kirikova M, Karagiannis D, Winter R (2013) BISE - Call for Papers Issue 1/2015. Advanced Enterprise Modeling. Bus Inf Syst Eng. doi: 10.1007/s12599-013-0289-x.

(C) Springer Fachmedien Wiesbaden 2013

\section{Schwerpunktthema}

Heutzutage nimmt die Geschwindigkeit von Veränderungen in der Wirtschaft stetig zu, während gleichzeitig der Kontext dieser Veränderungen zunehmend komplexer wird. Die globalisierte Wirtschaft erfordert zahlreiche Kooperatio- nen mit verschiedensten Organisationen und sich schnell ändernde Geschäftsaktivitäten und -prozesse in hochkompetitiven Umgebungen. Darum müssen Unternehmen in der Lage sein, schnell und effizient auf diese Veränderungen einzugehen und sie darüber hinaus sogar zum eigenen Wettbewerbsvorteil zu nutzen. Zu diesem Ziel wandeln Unternehmen sich stetig hin zu dynamischen, anpassungsfähigen und global integrierten Organisationen. Serviceorientierung und Cloud-Computing stellen zwei technologische Trends mit hohem Potenzial dar, um die genannten Herausforderungen zu bewältigen.

Forschung im Bereich Enterprise Modeling (EM) hat es sich zum Ziel gesetzt, ganzheitliche Lösungen für die Abstimmung von betriebswirtschaftlichen Zielen und IT zu finden, indem sie Techniken, Sprachen, Werkzeuge und Best Practices anbietet, um Modelle zur Repräsentation der Organisation und der Informationssysteme aus verschiedenen Perspektiven anzuwenden. Die obig beschriebenen komplexen unternehmerischen und technologischen Bedingungen verdeutlichen die Rolle des EM. Eigenschaften wie Agilität, Sensitivität, Reaktionsfreudigkeit, Anpassungsfähigkeit, Autonomie und Interoperabilität werden immer öfter als Normkomponenten für fortgeschrittene Unternehmensmodellen herangezogen. Diese ermöglichen es allen Einheiten eines Unternehmens, kooperativ zusammenzuwirken, um den Gesamtwert zu maximieren.

Das Schwerpunktheft möchte die unterstützenden Faktoren des EM erforschen, welche das Business-IT-Alignment in modernen Unternehmen fördern. Es dient als Forum für Forscher und Praktiker, sowohl hochqualitative Forschungsarbeiten als auch Fallstudien aus der Praxis in einer führenden internationalen Fachzeitschrift zu publizieren. Themengebiete umfassen unter anderem:

- Agile EM

- Anwendung der EM in unterschiedlichen organisationalen und technischen Kontexten
- Adaptionsfähigkeit von EM

- Übereinstimmung zwischen EM und Entwicklung von Informationssystemen

- EM-Sprachen, Referenzmodelle und Tools

- Evaluation von EM-Ansätzen, Erfolgsfaktoren in der EM

- Fallstudien und Praxis des EM

- EM-Management und -Steuerung

- Wiederverwendung von Unternehmensmodellen und Modellierungssprachen

\section{Einreichung von Beiträgen}

Bitte reichen Sie Beiträge für das Schwerpunktheft bis spätestens 2014-03-01 über das Online-Begutachtungssystem (http://www.editorialmanager.com/buis/) der Zeitschrift unter der Rubrik „BISE Advanced Enterprise Modeling“ ein. Bitte beachten Sie die Hinweise zu formaler Gestaltung und Umfang von Beiträgen für die WIRTSCHAFTSINFORMATIK/ Business \& Information Systems Engineering (BISE). Insbesondere sollten vollständige Beiträge maximal 50.000 Zeichen einschließlich Leerzeichen umfassen, abzüglich 5.000 Zeichen je Bild/Tabelle pro Seite. Ausführliche Autorenrichtlinien stehen unter http://www. wirtschaftsinformatik.de zum Download bereit.

Eingereichte Beiträge werden in einem doppelt-blinden Begutachtungsverfahren von mindestens drei Fachgutachtern auf Relevanz, Originalität und fachliche Qualität beurteilt.

\section{Zeitplan}

Einreichung der Beiträge bis: 2014-03-01 Benachrichtigung der Autoren: 2014-0426

Abschluss der ersten Überarbeitung: 2014-06-28

Abschluss der zweiten Überarbeitung (falls notwendig): 2014-09-20

Voraussichtliches Publikationsdatum: Januar 2015 